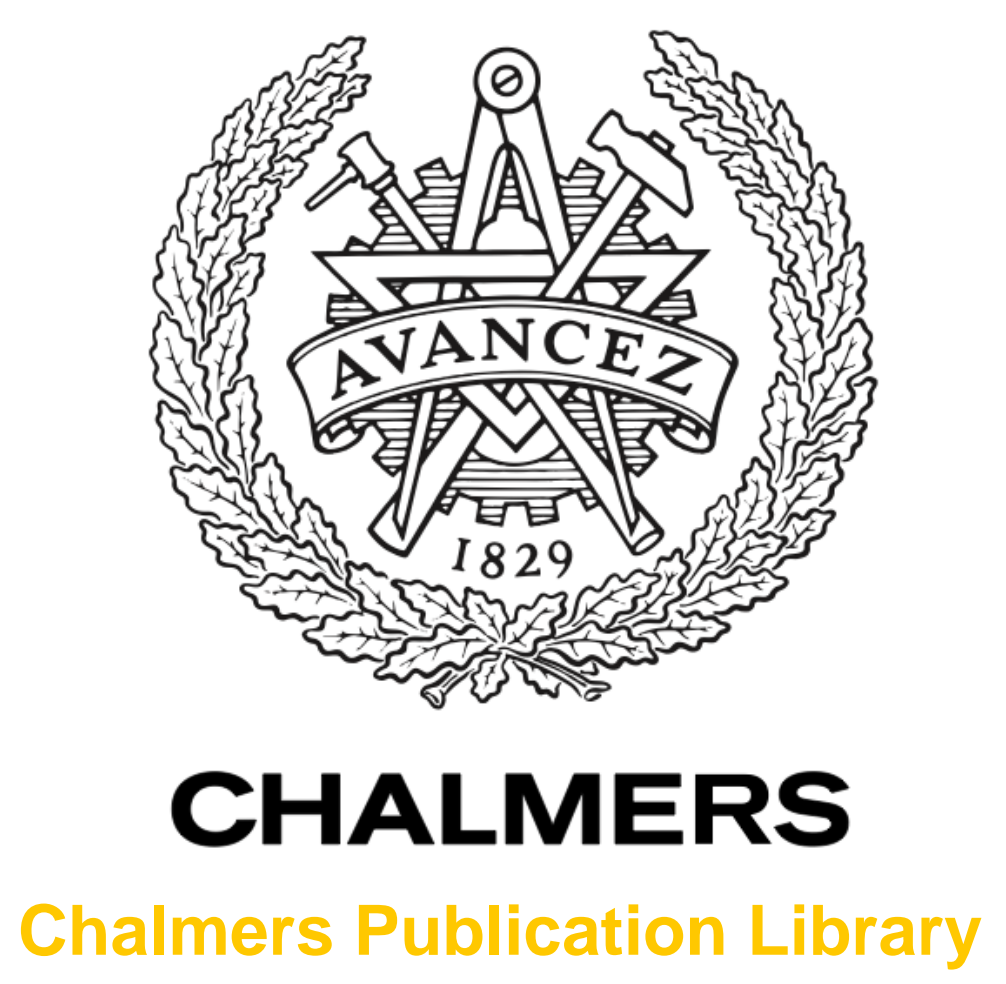

Performance Analysis and Cooperation Mode Switch in HARQ-based Relaying

This document has been downloaded from Chalmers Publication Library (CPL). It is the author's version of a work that was accepted for publication in:

IEEE Global Communications Conference (GLOBECOM) workshop, Austin, TX, December 2014

Citation for the published paper:

Li, J. ; Makki, B. ; Svensson, T. (2014) "Performance Analysis and Cooperation Mode

Switch in HARQ-based Relaying". IEEE Global Communications Conference

(GLOBECOM) workshop, Austin, TX, December 2014 pp. 930-935.

http://dx.doi.org/10.1109/GLOCOMW.2014.70635

Downloaded from: http://publications.lib.chalmers.se/publication/203337

Notice: Changes introduced as a result of publishing processes such as copy-editing and formatting may not be reflected in this document. For a definitive version of this work, please refer to the published source. Please note that access to the published version might require a subscription.

Chalmers Publication Library (CPL) offers the possibility of retrieving research publications produced at Chalmers University of Technology. It covers all types of publications: articles, dissertations, licentiate theses, masters theses, conference papers, reports etc. Since 2006 it is the official tool for Chalmers official publication statistics. To ensure that Chalmers research results are disseminated as widely as possible, an Open Access Policy has been adopted.

The CPL service is administrated and maintained by Chalmers Library. 


\title{
Performance Analysis and Cooperation Mode Switch in HARQ-based Relaying
}

\author{
Jingya Li, Behrooz Makki, and Tommy Svensson \\ Department of Signals and Systems, Chalmers University of Technology, Gothenburg, Sweden \\ Email: \{jingya.li, behrooz.makki, tommy.svensson\}@ chalmers.se
}

\begin{abstract}
We study the optimal, in terms of power-limited outage probability (OP), placement of the relay and investigate the effect of relay placement on the optimal cooperation mode of the source and the relay nodes. Using hybrid automatic repeat request (HARQ) based relaying techniques, general expressions for the OP and the average transmit power are derived. The results are then particularized to the repetition time diversity (RTD) protocol. The analytical expressions are used to find the transmit powers minimizing the power-limited OP. Our results demonstrate that adaptive power allocation reduces the $O P$ significantly. For instance, consider a Rayleigh fading channel, an OP of $10^{-3}$ and a maximum of 2 RTD-based retransmissions. Then, compared to equal power allocation, the required transmission signal-to-noise ratio (SNR) is reduced by $5 \mathrm{~dB}$, if adaptive power allocation is utilized. Another important observation is that, depending on the relay positions and the total power budget, the system should switch between the single-node transmission mode and the joint transmission mode, in order to minimize the outage performance.
\end{abstract}

\section{INTRODUCTION}

In future heterogeneous wireless networks, relay-assisted cooperative transmission is considered as one of the key techniques to improve the system reliability and ensure high quality of services [1], [2]. On the other hand, in current and future wireless communication networks, packet data transmission is based on hybrid automatic repeat request (HARQ) protocols. Utilizing forward error correction and error detection, HARQ techniques reduce the outage probability (OP) by retransmitting data that has experienced poor channel conditions. For this reason, a combination of relaying and HARQ protocols has received significant research attention for future wireless networks.

Based on the cooperation mode of the source and relay(s), HARQ-based relaying can be divided into two categories, namely, Single-Node Transmission (SNT) and multi-node Joint Transmission (JT). In the SNT mode, only one node (either the source or the relay) is active in each retransmission round. In the JT mode, once the relay decodes the data correctly, the source and the relay use, e.g., distributed spacetime coding to provide joint retransmission to the destination.

The performance of HARQ-based relaying was investigated in [3]-[10] and in [11]-[18], considering the SNT mode and the JT mode, respectively. However, it is not clear which

This work was supported in part by the Swedish Governmental Agency for Innovation Systems (VINNOVA) within the VINN Excellence Center Chase, and the Swedish Research Council VR under the project 621-20094555 Dynamic Multipoint Wireless Transmission.

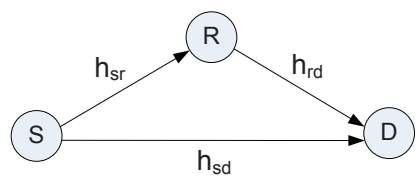

Figure 1. A relay-assisted communication system model.

cooperation mode is better. Moreover, the vast majority of existing works assume that all nodes have fixed powers for each (re)transmission round [4]-[20]. Retransmission power adjustment was investigated in [3] to improve the energy efficiency for basic HARQ schemes. However, the results in [3] are limited to the SNT cooperation mode.

Motivated by the above discussion, we hereafter study a cooperation mode switch in HARQ-based relaying, considering aptive power allocation. The OP is minimized under a total power constraint, which represents an important design factor as for the case of future green communications [21], [22]. The contributions of this paper are as follows:

- General expressions for the OP and the average total transmit power are derived, which apply to different HARQ-based relay networks with arbitrary transmit powers. The results are then particularized to the repetition time diversity (RTD) protocol.

- Retransmission power adjustment is used for minimizing the power-limited OP. We demonstrate that, compared to equal power allocation, with an OP of $10^{-3}$ and a maximum of 2 retransmissions, the average signal-tonoise ratio (SNR) is reduced by $5 \mathrm{~dB}$ in $\mathrm{RTD}$-based relaying if adaptive power allocation is utilized. Moreover, depending on the channel conditions and the total power budget, switching between the SNT mode and the JT mode is the optimal way for minimizing the OP.

- The optimal placement of the relay and the effect of the relay position on the optimal cooperation mode are investigated. We show that, in order for minimizing the power-limited OP the optimal relay position with equal power allocation is closer to the source. On the contrary, when performing adaptive power allocation, the optimal relay position is closer to the destination.

\section{SySTEM MODEL}

We consider a relay-assisted cooperative communication system comprising one source $(S)$, relay $(R)$ and destination node (D), as illustrated in Fig. 1. All nodes are equipped with a single antenna. Let $h_{\mathrm{sr}}, h_{\mathrm{rd}}$ and $h_{\mathrm{sd}}$ denote the channel 
coefficients of the S-to-R link, the R-to-D link, and the S-toD link, respectively. Correspondingly, we define the channel gains as $g_{\vartheta} \triangleq\left|h_{\vartheta}\right|^{2}$ with $\vartheta=\{\mathrm{sr}, \mathrm{rd}, \mathrm{sd}\}$. It is assumed that the channels are quasi-static, that is, the channel coefficients remain constant within a transmission block of $L_{c}$ channel uses, generally determined by the channel coherence time, and then change independently from one block to another according to their probability density functions (PDF).

An $H A R Q$-based retransmission protocol is utilized with a maximum of $M$ retransmission rounds, i.e., each codeword is (re)transmitted a maximum of $M+1$ times. Targeting for low-mobility users, the block length $L_{c}$ is assumed to be long such that all retransmission rounds occur in a single fading block. Define a packet as the subcodeword transmitted within a transmission block. Thus, the fading coefficients do not change during a packet period, and change independently from one packet to another. Moreover, the channel coefficient of each link is assumed to be perfectly known by it corresponding receiver. However, the transmitter of each link has no instantaneous channel state information (CSI), except for the HARQ feedback bits. It should be pointed out that, while we study the quasi-static model, it is not a necessary assumption and as shown in [23], there are mappings between the performance of HARQ protocols in quasi-static and fast-fading conditions.

In each transmission block, $S$ starts transmitting data to both $\mathrm{R}$ and $\mathrm{D}$. If the data is successfully decoded by $\mathrm{D}$, an acknowledgment (ACK) is fed back from D to both $\mathrm{S}$ and $\mathrm{R}$, then the retransmissions stop. Otherwise, D feeds back a negative-acknowledgment (NACK). R becomes active and feeds back an ACK to $\mathrm{S}$, as soon as it decodes the source message before $\mathrm{D}$. Once $\mathrm{R}$ is active, $\mathrm{S}$ and $\mathrm{R}$ provide joint data transmission to $\mathrm{D}$, and the data transmission continues until the data is correctly decoded by $\mathrm{D}$ or the maximum number of retransmissions is reached. Based on the feedback bits (ACK/NACK signals) from $\mathrm{R}$ and $\mathrm{D}$, the nodes $\mathrm{S}$ and $\mathrm{R}$ adapt their transmit powers so as to improve the OP. Note that, although the cooperation protocol allows joint transmission by $\mathrm{S}$ and $\mathrm{R}$, it may be optimal to keep the $\mathrm{S}$ off when the $\mathrm{R}$ decodes the data correctly. This point is further elaborated in the following.

\section{PROBLEM ForMULATION}

The following notation is used throughout the paper.

- $Q$ denotes the total number of information nats transmitted in each packet.

- $l$ represents the subcodeword length in each (re)transmission round. Thus, $r \triangleq Q / l$ (in natsper-channel-use (npcu)) gives the initial codeword rate.

- $P_{i 1}^{\mathrm{s}}$ and $P_{i 2}^{\mathrm{s}}$ denote the transmit power from $\mathrm{S}$ at the $i$-th round when $\mathrm{R}$ is inactive and active, respectively.

- $P_{i}^{\mathrm{r}}$ is the transmit power from $\mathrm{R}$ at the $i$-th round.

- $\operatorname{Pr}\left\{A_{m}\right\}$ denotes the probability of the event that the data is successfully decoded by $\mathrm{D}$ at the end of the $m$-th (re)transmission round while it was not decodable before.
In this case, the subcodewords may have been sent by $\mathrm{S}$ only or by $\mathrm{S}$ and $\mathrm{R}$ jointly.

- $\operatorname{Pr}\left\{A_{m, m}\right\}$ denotes the probability of the event that D successfully decodes the data at the end of the $m$ th (re)transmission round (and not before), when the codeword is only sent by $\mathrm{S}$, i.e. $\mathrm{R}$ is inactive in rounds $1, \ldots, m . \operatorname{Pr}\left\{A_{m, n}\right\}$ is the probability of the event that $\mathrm{D}$ successfully decodes the data at the end of the $m$-th (re)transmission round (and not before), when $\mathrm{R}$ is active in rounds $n+1, \ldots m$, with $n<m$.

- $\operatorname{Pr}\left\{B_{n}\right\}$ is the probability of the event that the data is successfully decoded by $\mathrm{R}$ at the end of the $n$-th (re)transmission round while it was not decodable before.

- $\operatorname{Pr}\left\{S_{m}\right\}$ is the probability of the event that data transmission stops at the end of the $m$-th (re)transmission round. In this case, either the maximum (re)transmission number, $M+1$, is reached, or the data is decoded by $\mathrm{D}$.

According to the definitions,

$$
\begin{aligned}
& \operatorname{Pr}\left\{A_{m}\right\}=\operatorname{Pr}\left\{A_{m, m}\right\}\left(1-\sum_{n=1}^{m-1} \operatorname{Pr}\left\{B_{n}\right\}\right) \\
&+\sum_{n=1}^{m-1} \operatorname{Pr}\left\{A_{m, n}\right\} \operatorname{Pr}\left\{B_{n}\right\} \\
& \operatorname{Pr}\left\{S_{m}\right\}= \begin{cases}\operatorname{Pr}\left\{A_{m}\right\}, & m=1, \ldots, M ; \\
1-\sum_{i=1}^{M} \operatorname{Pr}\left\{A_{i}\right\}, & m=M+1 .\end{cases}
\end{aligned}
$$

Our objective is to minimize the OP subject to an average total transmit power constraint $\phi_{\text {tot }}$. The target optimization problem can be written as

$$
\min _{\left\{P_{i 1}^{\mathrm{s}}, P_{i 2}^{\mathrm{s}}, P_{i}^{\mathrm{r}}\right\}} \psi, \quad \text { s.t. } \phi \leq \phi_{\text {tot }} .
$$

where $\psi$ denotes the $\mathrm{OP}$ and $\phi$ is the average total transmit power from $\mathrm{S}$ and $\mathrm{R}$. In order to formulate the optimization problem, we first derive general expressions for $\psi$ and $\phi$.

\section{A. Outage Probability}

The OP $\psi$ is defined as the probability that $\mathrm{D}$ cannot decode the data until the (re)transmission is stopped, that is,

$$
\psi=1-\sum_{m=1}^{M+1} \operatorname{Pr}\left\{A_{m}\right\} .
$$

Substituting (1) into (4), we get

$$
\begin{aligned}
\psi & =1-\sum_{m=1}^{M+1} \operatorname{Pr}\left\{A_{m, m}\right\} \\
& -\sum_{m=1}^{M+1} \sum_{n=1}^{m-1}\left(\operatorname{Pr}\left\{A_{m, n}\right\}-\operatorname{Pr}\left\{A_{m, m}\right\}\right) \operatorname{Pr}\left\{B_{n}\right\} .
\end{aligned}
$$

\section{B. Average Transmit Power}

The average total transmit power is defined as [24]

$$
\phi \triangleq \frac{\overline{\mathcal{E}}^{\mathrm{s}}+\overline{\mathcal{E}}^{\mathrm{r}}}{\overline{\mathcal{L}}}
$$

where $\overline{\mathcal{E}}^{\mathrm{s}}$ and $\overline{\mathcal{E}}^{\mathrm{r}}$ are the average energy of $\mathrm{S}$ and $\mathrm{R}$, averaged over many packet transmissions, respectively. Also, $\overline{\mathcal{L}}$ is the 
expected number of channel uses within each packet transmission period. If data transmission stops at the $m$-th round, the total number of channel uses is $\mathrm{ml}$. Therefore, the expected number of channel uses in each packet period is

$$
\begin{aligned}
\overline{\mathcal{L}} & =\left(\sum_{m=1}^{M+1} \operatorname{Pr}\left\{S_{m}\right\} m\right) l \\
& =\left(M+1-\sum_{m=1}^{M}(M+1-m) \operatorname{Pr}\left\{A_{m}\right\}\right) l .
\end{aligned}
$$

Next, we derive the expression for $\overline{\mathcal{E}}^{\mathrm{r}}$. If $\mathrm{R}$ correctly decodes the data at the end of the $n$-th round and data transmission stops at the end of the $m$-th round with $n<m$ (an event denoted by $B_{n} \& S_{m}$ ), the total energy consumed by $\mathrm{R}$ is $\mathcal{E}^{\mathrm{r}}=\sum_{i=n+1}^{m} P_{i}^{\mathrm{r}} l$. Hence, the consumed energy of the $\mathrm{R}$ is a random variable given by

$$
\mathcal{E}^{\mathrm{r}}=\sum_{i=n+1}^{m} P_{i}^{\mathrm{r}} l, \text { if } B_{n} \& S_{m}, m=1, \ldots, M+1, n<m .
$$

According to the definition,

$$
\begin{aligned}
& \operatorname{Pr}\left\{B_{n} \& S_{m}\right\} \\
& =\left\{\begin{array}{l}
\operatorname{Pr}\left\{A_{m, n}\right\} \operatorname{Pr}\left\{B_{n}\right\}, m=1, \ldots, M ; \\
\left(1-\sum_{i=1}^{n} \operatorname{Pr}\left\{A_{i, i}\right\}-\sum_{j=n+1}^{M} \operatorname{Pr}\left\{A_{j, n}\right\}\right) \operatorname{Pr}\left\{B_{n}\right\}, m=M+1 .
\end{array}\right.
\end{aligned}
$$

Therefore, the expected energy consumed by $\mathrm{R}$ is

$$
\begin{aligned}
\overline{\mathcal{E}}^{\mathrm{r}} & =\sum_{m=1}^{M} \sum_{n=1}^{m-1} \operatorname{Pr}\left\{A_{m, n}\right\} \operatorname{Pr}\left\{B_{n}\right\} \sum_{i=n+1}^{m} P_{i}^{\mathrm{r}} l \\
& +\sum_{n=1}^{M}\left(1-\sum_{i=1}^{n} \operatorname{Pr}\left\{A_{i, i}\right\}-\sum_{j=n+1}^{M} \operatorname{Pr}\left\{A_{j, n}\right\}\right) \operatorname{Pr}\left\{B_{n}\right\} \\
& \times \sum_{i=n+1}^{M+1} P_{i}^{\mathrm{r}} l
\end{aligned}
$$

The same procedure is applied to derive $\overline{\mathcal{E}}^{\text {s. }}$. If data transmission stops at the end of the $m$-th round while the codeword is only sent by $\mathrm{S}$, the total energy consumed by $\mathrm{S}$ is $\mathcal{E}^{\mathrm{s}}=\sum_{i=1}^{m} P_{i 1}^{\mathrm{s}} l$ for $m=1, \ldots, M+1$. If data transmission stops at the end of the $m$-th round while the codeword is jointly transmitted by $\mathrm{S}$ and $\mathrm{R}$ in rounds $n+1, \ldots m$, the total energy consumed by $\mathrm{S}$ is $\mathcal{E}^{\mathrm{s}}=\sum_{i=1}^{n} P_{i 1}^{\mathrm{s}} l+\sum_{i=n+1}^{m} P_{i 2}^{\mathrm{s}} l$ for $n<m$ and $m=2, \ldots, M+1$. Thus, the consumed energy of the $\mathrm{S}$ is a random variable given by

$\mathcal{E}^{\mathrm{s}}= \begin{cases}\sum_{i=1}^{m} P_{i 1}^{\mathrm{s}} l, & \text { if } \overline{B_{1} \& \ldots \& B_{m-1}} \& S_{m} \\ \sum_{i=1}^{n} P_{i 1}^{\mathrm{s}} l+\sum_{i=n+1}^{m} P_{i 2}^{\mathrm{s}} l, & \text { if } B_{n} \& S_{m}\end{cases}$

where

$$
\begin{aligned}
& \operatorname{Pr}\left\{\overline{\left.B_{1} \& \ldots \& B_{m-1} \& S_{m}\right\}}\right. \\
& = \begin{cases}\operatorname{Pr}\left\{A_{m, m}\right\}\left(1-\sum_{n=1}^{m-1} \operatorname{Pr}\left\{B_{n}\right\}\right), & m=1, \ldots, M \\
\left(1-\sum_{i=1}^{M} \operatorname{Pr}\left\{A_{i, i}\right\}\right)\left(1-\sum_{n=1}^{M} \operatorname{Pr}\left\{B_{n}\right\}\right), & m=M+1 .\end{cases}
\end{aligned}
$$

Therefore, the expected energy consumed by $\mathrm{S}$ is

$$
\begin{aligned}
\overline{\mathcal{E}}^{\mathrm{s}} & =\sum_{m=1}^{M} \operatorname{Pr}\left\{A_{m, m}\right\}\left(1-\sum_{n=1}^{m-1} \operatorname{Pr}\left\{B_{n}\right\}\right) \sum_{i=1}^{m} P_{i 1}^{\mathrm{s}} l \\
& +\sum_{m=1}^{M} \sum_{n=1}^{m-1} \operatorname{Pr}\left\{A_{m, n}\right\} \operatorname{Pr}\left\{B_{n}\right\}\left(\sum_{i=1}^{n} P_{i 1}^{\mathrm{s}}+\sum_{i=n+1}^{m} P_{i 2}^{\mathrm{s}}\right) l \\
& +\left(1-\sum_{m=1}^{M} \operatorname{Pr}\left\{A_{m, m}\right\}\right)\left(1-\sum_{n=1}^{M} \operatorname{Pr}\left\{B_{n}\right\}\right) \sum_{i=1}^{M+1} P_{i 1}^{\mathrm{s}} l \\
+ & \sum_{n=1}^{M}\left(1-\sum_{i=1}^{n} \operatorname{Pr}\left\{A_{i, i}\right\}-\sum_{j=n+1}^{M} \operatorname{Pr}\left\{A_{j, n}\right\}\right) \operatorname{Pr}\left\{B_{n}\right\} \\
& \times\left(\sum_{i=1}^{n} P_{i 1}^{\mathrm{s}}+\sum_{i=n+1}^{M+1} P_{i 2}^{\mathrm{s}}\right) l .
\end{aligned}
$$

Substituting (7), (10) and (11) into (6), and noting that $\psi$ can be expressed by (5), the average total transmit power is obtained as

$$
\phi=\frac{\varsigma}{M+1-\sum_{m=1}^{M}(M+1-m) \operatorname{Pr}\left\{A_{m}\right\}},
$$

where

$$
\begin{aligned}
\varsigma & =\sum_{m=1}^{M} \operatorname{Pr}\left\{A_{m, m}\right\}\left(1-\sum_{n=1}^{m-1} \operatorname{Pr}\left\{B_{n}\right\}\right) \sum_{i=1}^{m} P_{i 1}^{\mathrm{s}} \\
& +\sum_{m=1}^{M} \sum_{n=1}^{m-1} \operatorname{Pr}\left\{A_{m, n}\right\} \operatorname{Pr}\left\{B_{n}\right\}\left(\sum_{i=1}^{n} P_{i 1}^{\mathrm{s}}+\sum_{i=n+1}^{m}\left(P_{i 2}^{\mathrm{s}}+P_{i}^{\mathrm{r}}\right)\right) \\
& +\left(1-\sum_{m=1}^{M} \operatorname{Pr}\left\{A_{m, m}\right\}\right)\left(1-\sum_{n=1}^{M} \operatorname{Pr}\left\{B_{n}\right\}\right) \sum_{i=1}^{M+1} P_{i 1}^{\mathrm{s}} \\
& +\sum_{n=1}^{M}\left(1-\sum_{i=1}^{n} \operatorname{Pr}\left\{A_{i, i}\right\}-\sum_{j=n+1}^{M} \operatorname{Pr}\left\{A_{j, n}\right\}\right) \operatorname{Pr}\left\{B_{n}\right\} \\
& \times\left(\sum_{i=1}^{n} P_{i 1}^{\mathrm{s}}+\sum_{i=n+1}^{M+1}\left(P_{i 2}^{\mathrm{s}}+P_{i}^{\mathrm{r}}\right)\right) .
\end{aligned}
$$

It should be pointed out that the expressions for the OP and the average total transmit power, given in (5) and (12) respectively, are general for all HARQ protocols. The difference between different HARQ protocols and fading models is in the probability terms $\operatorname{Pr}\left\{A_{m, m}\right\}, \operatorname{Pr}\left\{A_{m, n}\right\}$ and $\operatorname{Pr}\left\{B_{n}\right\}$. In the following, as an example, we particularize the general problem formulation (3) to the RTD protocol. The same method can be applied for other HARQ protocols such as incremental redundancy and basic HARQ.

\section{Performance Analysis for the RTD Protocol}

From (3), it can be seen that, in order to formulate the optimization problems for the classical RTD protocol, we need to represent the probabilities $\left(\operatorname{Pr}\left\{A_{m, m}\right\}, \operatorname{Pr}\left\{A_{m, n}\right\}\right.$ and $\left.\operatorname{Pr}\left\{B_{n}\right\}\right)$ as functions of $P_{i 1}^{\mathrm{s}}, P_{i 2}^{\mathrm{s}}$ and $P_{i}^{\mathrm{r}}$ with $i=1, \ldots, M+$ 1. We consider independent Rayleigh fading channels, where the amplitudes of the communication links (i.e., $\left|h_{\mathrm{sr}}\right|,\left|h_{\mathrm{rd}}\right|$ and $\left.\left|h_{\mathrm{sd}}\right|\right)$ are independent non-identical Rayleigh distributed random variables. Thus, the channel gains (i.e., $g_{\vartheta}$ with 
$\vartheta=\{\mathrm{sr}, \mathrm{rd}, \mathrm{sd}\})$ follow the exponential distribution with PDF $f_{g_{\vartheta}}(x)=\lambda_{\vartheta} e^{-\lambda_{\vartheta} x}$ for $x>0$. The corresponding cumulative distribution function (CDF) is $F_{g_{\vartheta}}(x)=1-e^{-\lambda_{\vartheta} x}$ for $x>0$. Here, $\lambda_{\vartheta}=\frac{1}{\bar{g}_{\vartheta}}$ for $\vartheta=\{\mathrm{sr}, \mathrm{rd}, \mathrm{sd}\}$, where $\bar{g}_{\vartheta}$ is the expected channel gain determined by the pathloss and shadowing between the corresponding nodes. The noise powers at $\mathrm{R}$ and $\mathrm{D}$ are set to 1 .

In the RTD protocol, the same subcodeword is (re)transmitted in each round, and the received signals are combined by the receiver ( $\mathrm{R}$ and $\mathrm{D})$ using maximum ratio combining. Therefore, the received SNR at $\mathrm{R}$ after the $n$-th (re)transmission round increases to

$$
\gamma_{n}^{\mathrm{r}}=g_{\mathrm{sr}} \sum_{i=1}^{n} P_{i 1}^{\mathrm{s}}
$$

and the data rate reduces to $\frac{r}{n}$, where $r$ is the initial codeword rate defined in Section II. The data is successfully decoded by $\mathrm{R}$ at the end of the $n$-th round (and not before) if $\frac{r}{n-1}>\frac{1}{n-1} \log \left(1+\gamma_{n-1}^{\mathrm{r}}\right)$ and $\frac{r}{n} \leq \frac{1}{n} \log \left(1+\gamma_{n}^{\mathrm{r}}\right)$. Hence, the probability that $\mathrm{R}$ decodes the data at the end of round $n$, $\operatorname{Pr}\left\{B_{n}\right\}$, is

$$
\begin{aligned}
& \operatorname{Pr}\left\{B_{n}\right\} \\
& =\operatorname{Pr}\left\{\log \left(1+g_{\mathrm{sr}} \sum_{i=1}^{n-1} P_{i 1}^{\mathrm{s}}\right)<r \leq \log \left(1+g_{\mathrm{sr}} \sum_{i=1}^{n} P_{i 1}^{\mathrm{s}}\right)\right\} \\
& =\operatorname{Pr}\left\{\frac{e^{r}-1}{\sum_{i=1}^{n} P_{i 1}^{\mathrm{s}}} \leq g_{\mathrm{sr}}<\frac{e^{r}-1}{\sum_{i=1}^{n-1} P_{i 1}^{\mathrm{s}}}\right\} \\
& =e^{-\lambda_{\mathrm{sr}} \frac{e^{r}-1}{\sum_{i=1}^{n} P_{i 1}^{\mathrm{s}}}}-e^{-\lambda_{\mathrm{sr}} \frac{e^{r}-1}{\sum_{i=1}^{n-1} P_{i 1}^{\mathrm{s}}}} .
\end{aligned}
$$

The received SNR at $\mathrm{D}$ after the $m$-th (re)transmission round depends on whether the data is only sent by $\mathrm{S}$ or not. If $\mathrm{R}$ does not decode the data before the $m$-th round, the received SNR is

$$
\gamma_{m, m}^{\mathrm{d}}=g_{\mathrm{sd}} \sum_{i=1}^{m} P_{i 1}^{\mathrm{s}} .
$$

On the other hand, if $\mathrm{R}$ decodes the data at the end of $n$-th round, $n<m$, then $\mathrm{S}$ and $\mathrm{R}$ will provide joint transmission to $\mathrm{D}$ in rounds $n+1, \ldots, m$. Thus, the received SNR increases to

$$
\gamma_{m, n}^{\mathrm{d}}=g_{\mathrm{sd}}\left(\sum_{i=1}^{n} P_{i 1}^{\mathrm{s}}+\sum_{i=n+1}^{m} P_{i 2}^{\mathrm{s}}\right)+g_{\mathrm{rd}} \sum_{i=n+1}^{m} P_{i}^{\mathrm{r}} .
$$

Therefore, the probabilities $\operatorname{Pr}\left\{A_{m, m}\right\}$ and $\operatorname{Pr}\left\{A_{m, n}\right\}$ are found as

$$
\begin{aligned}
& \operatorname{Pr}\left\{A_{m, m}\right\} \\
& =\operatorname{Pr}\left\{\log \left(1+g_{\mathrm{sd}} \sum_{i=1}^{m-1} P_{i 1}^{\mathrm{s}}\right)<r \leq \log \left(1+g_{\mathrm{sd}} \sum_{i=1}^{m} P_{i 1}^{\mathrm{s}}\right)\right\} \\
& =\operatorname{Pr}\left\{\frac{e^{r}-1}{\sum_{i=1}^{m} P_{i 1}^{\mathrm{s}}} \leq g_{\mathrm{sd}}<\frac{e^{r}-1}{\sum_{i=1}^{m-1} P_{i 1}^{\mathrm{s}}}\right\} \\
& =e^{-\lambda_{\mathrm{sd}} \frac{e^{r}-1}{\sum_{i=1}^{m} P_{i 1}^{\mathrm{s}}}}-e^{-\lambda_{\mathrm{sd}} \frac{e^{r}-1}{\sum_{i=1}^{m-1} P_{i 1}^{\mathrm{s}}}}
\end{aligned}
$$

and

$$
\begin{aligned}
& \operatorname{Pr}\left\{A_{m, n}\right\} \\
& =\operatorname{Pr}\left\{\log \left(1+g_{\mathrm{sd}}\left(\sum_{i=1}^{n} P_{i 1}^{\mathrm{s}}+\sum_{i=n+1}^{m-1} P_{i 2}^{\mathrm{s}}\right)+g_{\mathrm{rd}} \sum_{i=n+1}^{m-1} P_{i}^{\mathrm{r}}\right)\right. \\
& \left.<r \leq \log \left(1+g_{\mathrm{sd}}\left(\sum_{i=1}^{n} P_{i 1}^{\mathrm{s}}+\sum_{i=n+1}^{m} P_{i 2}^{\mathrm{s}}\right)+g_{\mathrm{rd}} \sum_{i=n+1}^{m} P_{i}^{\mathrm{r}}\right)\right\} \\
& =e^{-\frac{\lambda_{\mathrm{r}}\left(e^{r}-1\right)}{\sum_{i=n+1}^{\mathrm{d}} P_{i}^{\mathrm{r}}}}-e^{-\frac{\lambda_{\mathrm{rd}}\left(e^{r}-1\right)}{\sum_{i=n+1}^{m-1} P_{i}^{\mathrm{r}}}}+K_{1}+K_{2}
\end{aligned}
$$

respectively, where

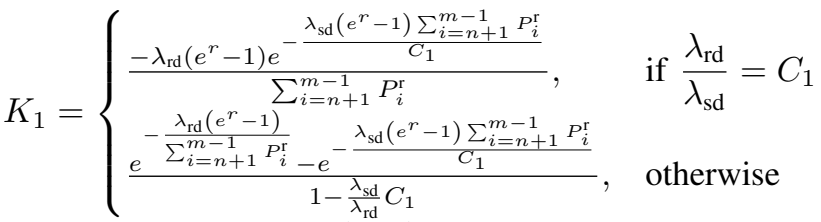

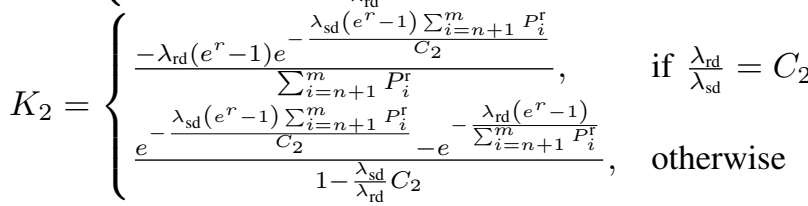

with $C_{1} \triangleq \frac{\sum_{i=n+1}^{m-1} P_{i}^{\mathrm{r}}}{\sum_{i=1}^{n} P_{i 1}^{\mathrm{s}}+\sum_{i=n+1}^{m-1} P_{i 2}^{\varsigma}}, C_{2} \triangleq \frac{\sum_{i=n+1}^{m} P_{i}^{\mathrm{r}}}{\sum_{i=1}^{n} P_{i 1}^{\S}+\sum_{i=n+1}^{m} P_{i 2}^{\varsigma}}$.

Substituting the probability terms $\operatorname{Pr}\left\{A_{m, m}\right\}, \operatorname{Pr}\left\{A_{m, n}\right\}$ and $\operatorname{Pr}\left\{B_{n}\right\}$ into (5) and (12), closed-form expressions for the OP and the average total transmit power, i.e., the optimization parameters of problem 3 , are derived for the considered RTD protocol, as functions of $P_{i 1}^{\mathrm{s}}, P_{i 2}^{\mathrm{s}}$ and $P_{i}^{\mathrm{r}}, i=1, \ldots, M+1$. Then, from (3), the optimization problem can be formulated.

\section{Simulation Results and Discussions}

In this section, we evaluate the performance of the RTDbased relaying with adaptive power allocation under powerlimited conditions. The optimization problem is nonconvex in general and difficult to solve [12]. However, our analytical expressions obtained in Sections III and IV make it possible to find the (sub)optimal power allocation solutions and evaluate the system performance numerically. As the problem is nonconvex, we cannot guarantee that our results are globally optimal. For this reason, we have checked our results with different methods such as the iterative algorithm of [25], and the "fminsearch" and "fmincon" functions of MATLAB. In all cases, the same results are obtained which is an indication of a reliable result. The performance of the optimal adaptive power allocation schemes are compared with an equal power allocation scheme where $P_{i 1}^{\mathrm{s}}=P_{i 2}^{\mathrm{s}}=P_{i}^{\mathrm{r}}$ for $\forall i$.

\section{A. On the Impact of Adaptive Power Allocation}

1) Outage Probability Analysis: The OP of the RTD-based relaying system is plotted in Fig. 2 as a function of the average total transmit power for $M=1$ and $M=2$ retransmissions, i.e., 2 and 3 (re)transmissions. We see that, compared to the equal power allocation scheme, adaptive power allocation reduces the OP considerably when the total transmit power budget is large. Moreover, for a given average total transmit power, increasing the number of retransmissions $M$ leads to 


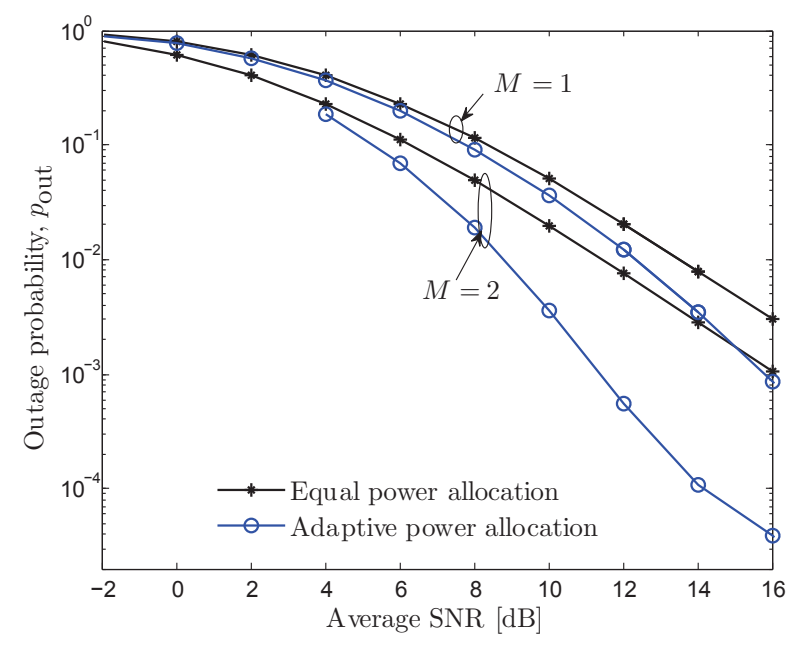

Figure 2. Outage probability $p_{\text {out }}$ vs. the average total transmit power $\phi_{\text {tot }}$, with $r=1, \lambda_{\mathrm{sd}}=2$, and $\lambda_{\mathrm{sr}}=\lambda_{\text {rd }}=1$.

substantial outage performance gain for the adaptive power allocation scheme. For instance, consider an OP of $10^{-3}$ and a maximum of 2 RTD-based retransmissions. Then, compared to equal power allocation, the required transmission SNR is reduced by $5 \mathrm{~dB}$, if adaptive power allocation is utilized. This is because with more number of retransmissions $\mathrm{R}$ gets more involved and the good characteristics of R-to-D link are properly exploited.

2) Cooperation Mode Switch: Figure 3 investigates the cooperation mode switch with adaptive power allocation for the RTD-based relaying. The optimal power values are plotted as a function of $\phi_{\text {tot }}$. The expected channel gains are set to $\bar{g}_{\mathrm{sd}}=0.5$ and $\bar{g}_{\mathrm{sr}}=\bar{g}_{\mathrm{rd}}=1$. From Fig. 3 we see that $P_{22}^{\mathrm{s}}=0$ when $\phi_{\text {tot }}$ is small. When $\phi_{\text {tot }}>6 \mathrm{dBm}, P_{22}^{\mathrm{s}}$ becomes larger than zero and it increases as $\phi_{\text {tot }}$ increases. This implies that when the total transmit power budget is small, the SNT mode is optimal for the RTD-based relaying. As the total transmit power becomes large, the RTD-based relaying system should switch to the JT mode in order to minimize the OP. Moreover, as anticipated, when $\mathrm{R}$ is active, the transmit power from the $\mathrm{R}$ node is always larger than the transmit power from the $\mathrm{S}$, i.e., $P_{2}^{\mathrm{r}}>P_{22}^{\mathrm{s}}$. This is because, with the considered values of $\lambda_{\mathrm{sr}}, \lambda_{\mathrm{rd}}$ and $\lambda_{\mathrm{sd}}$, the R-to-D channel has a better average channel characteristics compared to the S-to-D channel, i.e., $\bar{g}_{\text {rd }}>\bar{g}_{\text {sd }}$. This point is summarized in Theorem 1 as follows.

Theorem 1: Under an average total transmit power constraint, there exist a threshold $\phi_{\text {th }}$, such that the SNT mode is optimal in terms of OP if $\phi_{\text {tot }}<\phi_{\mathrm{th}}$. On the other hand, when $\phi_{\text {tot }} \geq \phi_{\text {th }}$, the JT mode becomes the optimal cooperation mode in terms of outage-limited average power.

Proof: The proof follows from the same concept as in the water-filling technique where the better channels receive more power. Without loss of generality, we assume that $\bar{g}_{\text {rd }}>\bar{g}_{\text {sd }}$, i.e., the R-to-D channel has a better average channel characteristics than the S-to-D channel. Then, once $\mathrm{R}$ decodes the data, it is always better to allocate more transmit power to $R$ (i.e., the node which is more likely to see a better channel to the D). When the total power budget $\phi_{\text {tot }}$ is small, the water-

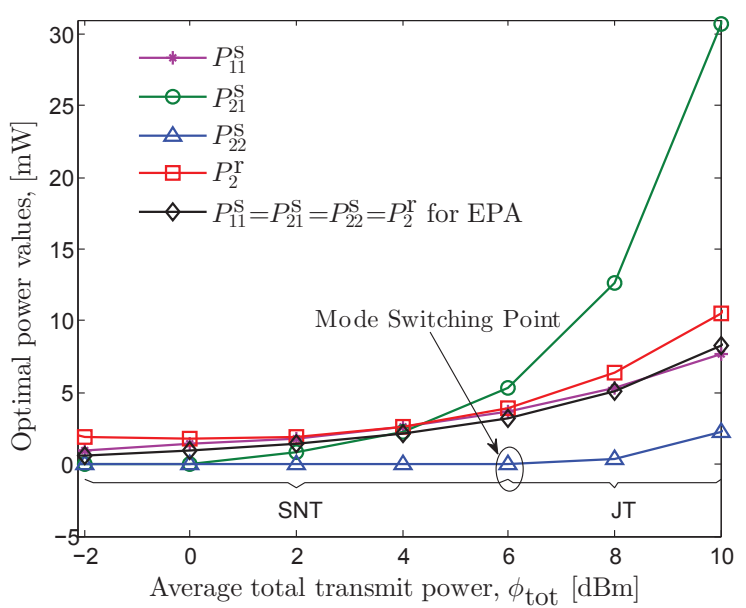

Figure 3. Optimal power values vs. the average total transmit power $\phi_{\text {tot }}$, with $r=1, M=1, \lambda_{\mathrm{sd}}=2$, and $\lambda_{\mathrm{sr}}=\lambda_{\mathrm{rd}}=1$.

level is low. Thus, all the transmit power will be allocated to $\mathrm{R}$, while the transmit power from $\mathrm{S}$ (i.e., the node associated to the weaker channel) is zero. As the total power budget $\phi_{\text {tot }}$ increases, the water-level increases. Therefore, both $\mathrm{R}$ and $\mathrm{S}$ become active and the relay network exploits the cooperative diversity. In this case, the JT mode becomes optimal in order to achieve better diversity gain.

\section{B. On the Impact of the $R$ Position}

We consider a setup where $\mathrm{R}$ is located on the line between $\mathrm{S}$ and $\mathrm{D}$. The position of $\mathrm{R}$ is identified by the ratio $\delta \triangleq \frac{d_{\mathrm{sr}}}{d_{\mathrm{sd}}}$, where $d_{\text {sr }}$ and $d_{\text {sd }}$ denote the distances between $\mathrm{S}$ and $\mathrm{R}$, and $\mathrm{S}$ and $\mathrm{D}$, respectively. The expected channel gains are modeled by $\bar{g}_{\vartheta}=\alpha_{\vartheta}\left(d_{\vartheta}\right)^{-\beta_{\vartheta}}$ for $\vartheta=\{\mathrm{sr}, \mathrm{rd}, \mathrm{sd}\}$, where $\beta_{\vartheta}$ is the path loss exponent of the corresponding link, and $\alpha_{\vartheta}$ is a parameter independent of $d_{\vartheta}$ and contains parameters such as the transmit antenna gain, receive antenna gain and shadowing. Here, we set $\beta_{\mathrm{sr}}=\beta_{\mathrm{sd}}=\beta_{\mathrm{rd}}=3, \alpha_{\mathrm{sr}}=\alpha_{\mathrm{sd}}=\alpha_{\mathrm{rd}}$ and $\bar{g}_{\text {sd }}=1$. We consider the RTD-based relaying with $M=1$ and $r=1$. The (re)transmission powers for each node are optimized to minimize the OP $P_{\text {out }}$, subject to an average total power constraint $\phi_{\text {tot }}=10 \mathrm{dBm}$.

1) Outage Probability Analysis: Figure 4 illustrates the $\mathrm{OP}$ as a function of the distance ratio. It can be seen that, compared to equal power allocation, adaptive power allocation can reduce the OP substantially, especially when the distance ratio is large, i.e., when $\mathrm{R}$ is placed closer to $\mathrm{D}$. We also observe that there exists an optimal relay position that minimizes the OP. The optimal relay position with equal power allocation is closer to S. While when performing adaptive power allocation, the optimal relay position is closer to D. This observation is intuitive, since as the distance ratio $\delta$ increases, i.e., when the $\mathrm{R}$ moves towards the $\mathrm{D}$, the R-to-D link becomes much better than the S-to-D link on average. Thus, once the $\mathrm{R}$ decodes the data, the system should switch to the SNT mode, i.e., the $\mathrm{S}$ should be switched off while all power should be allocated to the R (see Fig. 4). For the equal power allocation scheme, the transmit powers allocated to the $\mathrm{S}$ and $\mathrm{R}$ are always the same, thus, the optimal relay position should be closer to $S$, 


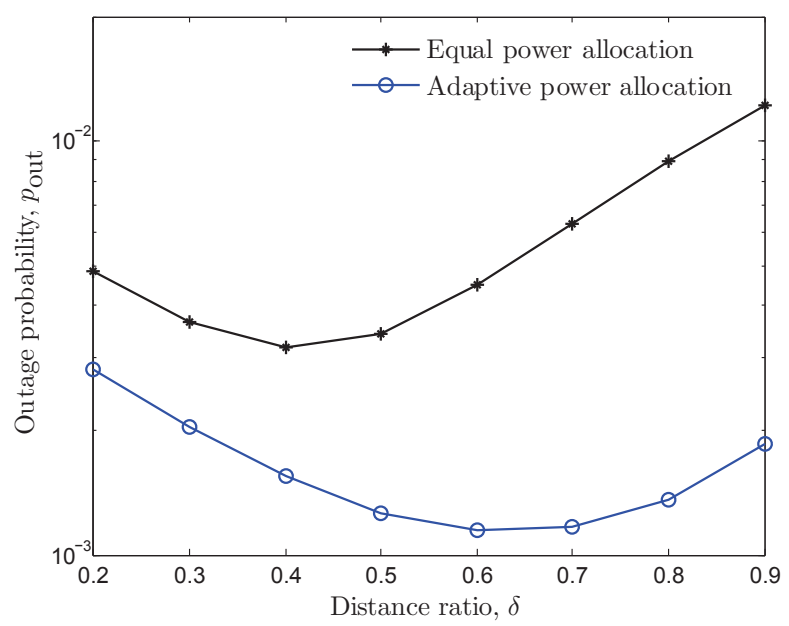

Figure 4. Outage probability $p_{\text {out }}$ vs. the distance ratio $\delta$.

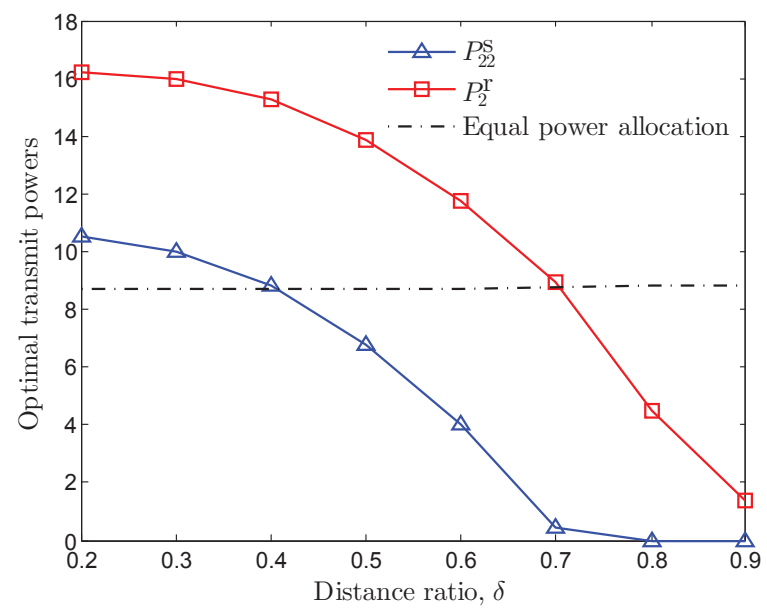

Figure 5. Optimal transmit powers, $P_{22}^{S}$ and $P_{2}^{R}$, vs. the distance ratio $\delta$.

in order to gain more benefits from the S-to-D link.

2) Cooperation Mode Switch: Finally, we investigate the effects of $\mathrm{R}$ position on the cooperation mode selection. Figure 5 plots the optimal transmit powers, $P_{22}^{\mathrm{s}}$ and $P_{2}^{\mathrm{r}}$, of the adaptive power allocation scheme and the power values of the equal power allocation scheme used in Fig. 4. We see that when $\delta \geq 0.8, P_{22}^{\mathrm{s}}$ becomes zero, i.e., $\mathrm{S}$ stops retransmission when $\mathrm{R}$ is active. This implies that when the average channel gain difference between R-to-D link and S-to-D link, $\bar{g}_{\text {rd }}-\bar{g}_{\text {sd }}$, is small, the JT mode is optimal. However, as $\bar{g}_{\mathrm{rd}}-\bar{g}_{\mathrm{sd}}$ becomes large, the system should switch to the SNT mode in order to minimize the OP.

\section{CONCLUSION}

In this paper, cooperation mode switch was investigated in relay-HARQ networks by using adaptive power allocation. In particular, analytical expressions for the OP and the average total transmit power have been derived for the RTD protocol. Retransmission power adjustment is used for minimizing the OP of the network, subject to an average total transmit power constraint. We showed that adaptive power allocation can significantly reduce the OP. Moreover, it is always better to select the SNT mode when the total power budget is low, or when the average channel gain difference between the R-to-D link and the S-to-D link is large. However, as the total transmit power increases or the average channel gain difference decreases, the system should switch to the JT mode. Finally, the optimal cooperation mode is remarkably affected by the relay position.

\section{REFERENCES}

[1] C. Hoymann et al., "Relaying operation in 3GPP LTE: Challenges and solutions," IEEE Commun. Mag., vol. 50, no. 2, pp. 156-162, June 2012.

[2] EU FP7 INFSO-ICT-317669 METIS, D3.2, "First performance results for multi-node/multi-antenna transmission technologies," Apr. 2014.

[3] H. Seo and B. G. Lee, "Optimal transmission power for single- and multi-hop links in wireless packet networks with ARQ capability," IEEE Trans. Commun., vol. 55, no. 5, pp. 996-1006, May 2007.

[4] J. Park and J. H. Lee, "Effect of outdated CSI on the performance of opportunistic relaying with ARQ," in VTC, Sept. 2012.

[5] S. H. Kim et al., "Optimal rate selection scheme in a two-hop relay network adopting chase combining HARQ in rayleigh block-fading channels," in WCNC, April 2012.

[6] J. Choi, D. To, Y. Wu, and S. Xu, "Energy-delay tradeoff for wireless relay systems using HARQ with incremental redundancy," IEEE Trans. Wireless Commun., vol. 12, no. 2, pp. 561-573, Feb. 2013.

[7] J. Choi et al., "On the energy efficiency of a relaying protocol with HARQ-IR and distributed cooperative beamforming," IEEE Trans. Wireless Commun., vol. 12, no. 2, pp. 769-781, Feb. 2013.

[8] G. Yu et al., "Efficient ARQ protocols for exploiting cooperative relaying in wireless sensor networks," Computer Commun., vol. 30, no. 14-15, pp. $2765-2773$, Oct. 2007.

[9] G. Choi, W. Zhang, and X. Ma, "Achieving joint diversity in decodeand-forward MIMO relay networks with zero-forcing equalizers," IEEE Trans. Commun., vol. 60, no. 6, pp. 1545-1554, June 2012.

[10] P. Zhang et al., "Joint power allocation and relay selection for multi-hop cognitive network with ARQ," in PIMRC, Sept. 2012.

[11] I. Stanojev et al., "Energy efficiency of non-collaborative and collaborative hybrid-ARQ protocols," IEEE Trans. Wireless Commun., vol. 8, no. 1, pp. 326-335, Jan. 2009.

[12] S. Lee et al., "The optimal power assignment for cooperative hybridARQ relaying protocol," in GLOBECOM, Dec. 2011.

[13] N. Abuzainab and A. Ephremides, "Energy efficiency of cooperative relaying over a wireless link," IEEE Trans. Wireless Commun., vol. 11, no. 6, pp. 2076-2083, June 2012.

[14] Y. Qi, R. Hoshyar, M. Imran, and R. Tafazolli, "The energy efficiency analysis of HARQ in hybrid relaying systems," in VTC, May 2011.

[15] —_ "H2-ARQ-relaying: Spectrum and energy efficiency perspectives," IEEE J. Sel. Areas Commun., vol. 29, no. 8, pp. 1547-1558, Sept. 2011.

[16] B. Maham, A. Behnad, and M. Debbah, "Analysis of outage probability and throughput for half-duplex hybrid-ARQ relay channels," IEEE Trans. Veh. Technol., vol. 61, no. 7, pp. 3061-3070, Sept. 2012.

[17] I. Stanojev et al., "Performance analysis of collaborative hybrid-ARQ incremental redundancy protocols over fading channels," in SPAWC, July 2006.

[18] R. Narasimhan, "Throughput-delay performance of half-duplex hybridARQ relay channels," in ICC, May 2008, pp. 986-990.

[19] I. Stanojev, O. Simeone, Y. Bar-Ness, and C. You, "Performance of multi-relay collaborative hybrid-ARQ protocols over fading channels," IEEE Commun. Lett., vol. 10, no. 7, pp. 522-524, July 2006.

[20] I. Byun et al., "Performance analysis of a decode-and-forward based hybrid-ARQ protocol," in MILCOM, Nov. 2008.

[21] Y. Chen et al., "Fundamental trade-offs on green wireless networks," IEEE Commun. Mag., vol. 49, no. 6, pp. 30-37, June 2011.

[22] Z. Hasan et al., "Green cellular networks: A survey, some research issues and challenges," IEEE Commun. Surveys Tut., vol. 13, no. 4, pp. 524540, Nov. 2011.

[23] B. Makki et al., "On noisy ARQ in block-fading channels," IEEE Trans. Veh. Technol., vol. 63, no. 2, pp. 731-746, Feb 2014.

[24] G. Caire et al., "Optimum power control over fading channels," IEEE Trans. Inf. Theory, vol. 45, no. 5, pp. 1468-1489, Jul. 1999.

[25] B. Makki and T. Eriksson, "On hybrid ARQ and quantized CSI feedback schemes in quasi-static fading channels," IEEE Trans. Commun., vol. 60 , no. 4, pp. 986-997, April 2012. 